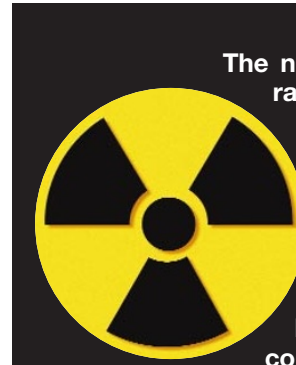

The nuclear arms race generated thousands of disposal sites containing radioactive toxic wastes dumped into the ground. Bioremediation is regarded as a relatively cheap and effective way of cleaning up these sites; however, this will require microorganisms that can detoxify heavy-metal and organic pollutants while withstanding high doses of radiation. In this issue Brim et al. have demonstrated the feasibility of this strategy by genetically engineering microbes that can detoxify radioactive wastes with mixed compositions. They started with Deinococcus radiodurans - the most radiation-resistant organism yet known, and engineered it to express an Escherichia coli enzyme that converts ionic mercury to a less toxic form, and a Pseudomonas enzyme that breaks down toluene (see page 85).

\section{Screening for site-specific proteases}

Site-specific proteases are key players in the etiology of certain diseases-Alzheimer's being a prime example. In this issue, Döbeli and colleagues describe a generally applicable and high-throughput assay system for detecting site-specific proteases from crude cell homogenates (see p. 66). The protein substrate is coupled to ceramic beads and incubated with cell homogenates; then the peptides are washed, released, and analyzed by mass spectrometry, allowing for purification of proteases that clip a protein at the site of interest.

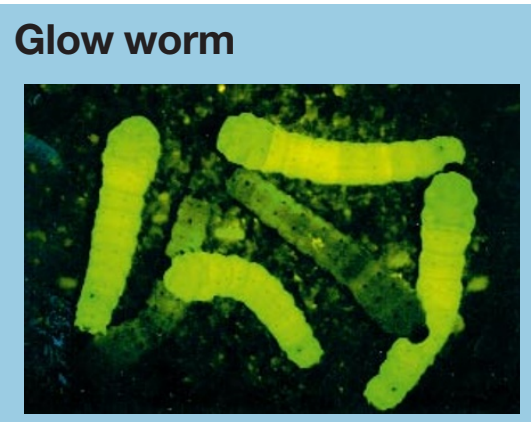

Although the silkworm Bombyx mori is an insect of great economic importance, it has until now resisted efforts for efficient germline transgenesis, most likely because the usual insect transgenesis strategies rely on transposons that do not function in lepidopteran insects like silkworms. Toshik et al. now have succeded in silkworm transgenesis using piggyBac, a transposon discovered in another lepidopteran species. In this study, they transformed the silkworms with GFP and got glowing green worms. One can easily imagine more pragmatic applications, however, like engineering silk glands for production of novel fibers or proteins of pharmaceutical interest (see p. 81).

Research briefs written by Natalie DeWitt and Robert Frederickson

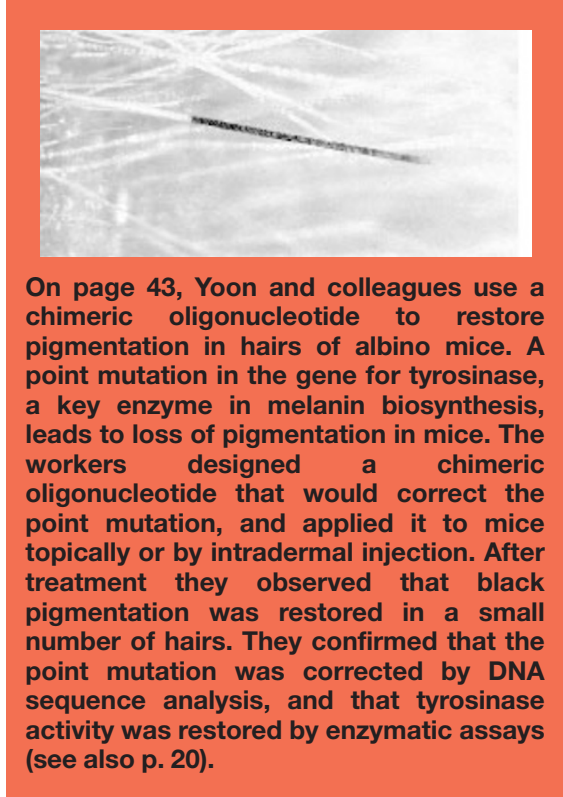

\section{A new array of possibilities}

The development of rapid, cost-effective strategies to screen for disease-associated genetic mutations is an important goal of molecular diagnostics. Although DNA chips are powerful tools for high-throughput, parallel sequence analysis, efforts are being undertaken to develop alternative technologies. On page 91, Walt and colleagues demonstrate the application of their fiber-optic gene array technology into a platform for the analysis of cystic fibrosis related mutations. Their array comprises a collection of chemically encoded microspheres linked to individual molecular beacon probes, which have been distributed in tiny wells at the tip of individual optical fibers. Specific advantages of their system include the elimination of the need for target labeling, and the capacity for parallel analysis of subnanomolar concentrations of target DNA.

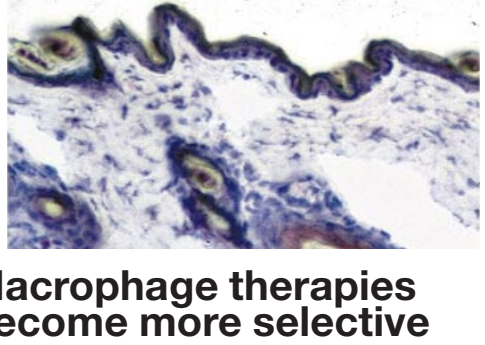

Macrophages are multifunctional immune cells that play important regulatory roles in the skin's response to inflammatory agents. This has led to the suggestion that elimination of these cells could be beneficial in the treatment of chronic inflammatory dermatoses of the skin. Although systemic elimination of macrophage function has been shown to have therapeutic potential, it is often associated with general immunosuppression. To avoid this problem, Thepen et al. have targeted a specific local subset of macrophages, based on their enhanced expression of an immunoglobulin receptor during the inflammatory response. By injecting a toxin linked to an antibody that binds the macrophage receptors, they were able to demonstrate local elimination of the macrophages and resolution of the inflammatory response in a mouse model system (see p. 25 and 48).

\section{Longer life in vitro with telomerase}

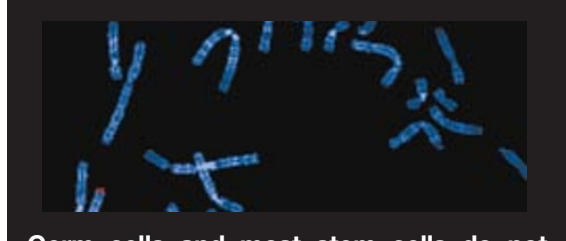

Germ cells and most stem cells do not suffer the progressive shortening of telomere length that can limit the proliferative capacity of many somatic cells in vitro. Ectopic expression of telomerase reverse transcriptase (TERT), a ribonucleoprotein complex that adds telomeric DNA to the ends of chromosomes, has been shown to extend the proliferative potential of certain somatic cells. The use of TERT overexpression to enhance the culture lifespan of somatic cells for tissue engineering purposes requires that the process not lead to tumorigenic potential or disturb the differentiated cell phenotype. On page 39 , Thomas et al. demonstrate that TERT expression in bovine adrenocortical cells extends their lifespan without affecting their capacity to form functional adrenal tissue after transplantation into adrenalectomized immunodeficient mice (see also p. 22). 